\title{
O PROBLEMA DA CONTAMINAÇÃo NA DETERMINAÇÃo DE TRAÇOS DE ALUMÍNIO
}

\author{
Solange Cristina Garcia, Adriana Gioda e Denise Bohrer do Nascimento*
}

Departamento de Química - Universidade Federal de Santa Maria - UFSM - 97119-000 - Santa Maria - RS

Recebido em 13/5/96; aceito em 11/10/96

\begin{abstract}
THE PROBLEM OF CONTAMINATION IN THE ALUMINIUM TRACE ANALYSIS. The most common difficulties which occur in trace analysis are those which involve contamination, mainly when the measurement is at very low concentration of ubiquitous elements as aluminium. The worst situation is when a separation step is necessary, because it requires extra-manipulations of the sample. This article describes the degree of contamination and its control for the aluminium trace analysis in dialysis solutions, when aluminium content of about $15 \mu \mathrm{g} / \mathrm{l}$ must be determinated.
\end{abstract}

Keywords: trace analysis; contamination; aluminium.

\section{INTRODUÇÃO}

$\mathrm{Na}$ determinação de traços, a contaminação parece ser o principal fator limitante quando se busca determinar quantidades muito pequenas de um analito em amostras reais.

Podem contribuir para a contaminação além dos reagentes e recipientes utilizados na análise, pó, fumaça, partículas provenientes de paredes, chão, teto, roupas e de materiais naturais como, por exemplo o pólen.

O controle da contaminação torna-se particularmente difícil quando o elemento que se quer determinar a nível de traço é onipresente no meio ambiente. No caso particular do alumínio, que é o terceiro elemento mais abundante da crosta terrestre, observa-se que ele atinge a atmosfera não só através de emissões naturais, mas principalmente antropogênicas em função do seu grande emprêgo a nível industrial. Além do uso geral do alumínio como metal, o óxido de alumínio é componente de quase todos os tipos de vidros, e compostos de alumínio são utilizados como catalisadores na fabricação de plásticos.

$\mathrm{O}$ uso de sulfato de alumínio como agente floculante em estações de tratamento d'água representa uma fonte adicional de contaminação, uma vez que traços de alumínio existentes na água não são totalmente eliminados pelos processos convencionais de purificação - destilação e troca-iônica - usuais em laboratórios.

\section{A IMPORTÂNCIA DA DETERMINAÇÃO DE ALUMÍNIO A NÍVEL DE TRAÇOS}

Dentro da química analítica do alumínio dois capítulos distintos devem ser considerados. Um deles trata da análise de ligas metálicas, solos, águas e vegetais, onde via de regra, o conteúdo em alumínio situa-se na faixa de partes por milhão $(\mathrm{ppm})$, e problemas de contaminação não chegam a ser críticos. O outro trata da determinação de alumínio em fluídos para hemodiálise e no organismo humano, onde a quantidade de alumínio não deve ultrapassar poucas partes por bilhão $(\mathrm{ppb})^{1}$.

Como o alumínio não é um elemento essencial ao corpo humano, sua importância reside no efeito tóxico e cumulativo que provoca em pacientes com insuficiência renal crônica (IRC), que necessitam submeter-se periodicamente à hemodiálise. Pessoas com IRC submetem-se três vezes por semana a sessões de hemodiálise que duram de 3 a 4 horas. Para ser purificado, o sangue do paciente interage com com a solução de diálise, aproximadamente 120 litros de água na qual é dissolvido um concentrado salino, geralmente constituído por cloretos de sódio, potássio, cálcio e magnésio, acetato de sódio e ainda glicose, dextrose e/ou lactose ${ }^{2}$. Sangue e solução de diálise circulam pelos dois lados da membrana dialisadora, de tal forma que as impurezas passam do sangue para a solução de diálise sem que contudo sejam dialisados os seus principais eletrólitos. Quando ocorre da água estar contaminada por alumínio, este faz o caminho inverso. Entrando na corrente sanguínea os íons alumínio acumulam-se no organismo por deposição nos ossos e no cérebro provocando distrofia óssea e distúrbios neurológicos ${ }^{3}$. Há na IUPAC um sub-comitê da Comissão de Toxicologia encarregado do controle do alumínio em soluções de diálise ${ }^{4}$. Este subcomitê fixou em $15 \mu \mathrm{g} / \mathrm{l}$ a concentração máxima de alumínio em soluções de diálise. Isto impõe a necessidade de um controle de qualidade muito rigoroso tanto para a água de hemodiálise quanto para o concentrado salino utilizado no preparo da solução.

\section{DETERMINAÇÃO DE ALUMÍNIO EM FLUIDOS PARA HEMODIÁLISE E AMOSTRAS BIOLÓGICAS}

Para uma determinação segura de quantidades tão pequenas, considerando a complexidade das matrizes, a sensibilidade da maioria dos métodos não permite uma determinação direta. A técnica mais empregada neste tipo de análise é a espectrometria de absorção atômica (AAS) em forno de grafite $^{5}$, com a qual, via de regra, podem-se medir diretamente baixas concentrações. Mas, tanto para os fluidos de diálise quanto para amostras biológicas, mais especificamente o soro sangüíneo, uma determinação direta não é possível (a concentração normal de alumínio no sangue situa-se em torno de $7 \mu \mathrm{g} / \mathrm{l}^{6}$ ). Devido à sua alta concentração salina, os fluidos costumam ser diluídos muitas vezes, encontrando-se na literatura diluições em até 37 vezes $^{7}$. No caso do soro diluições de no mínimo 1:1 são usuais ${ }^{8}$.

Via de regra estas diluições, tendo como objetivo a determinação por AAS, utilizam não apenas água como diluente mas também soluções de ácidos como ácido nítrico ${ }^{9,10}$, tensoativos como Triton $^{11}$, misturas de ambos ${ }^{12-14}$, ou ainda modificadores químicos adequados à determinação de alumínio, encontrandose na literatura, além do clássico nitrato de magnésio ${ }^{15}$, outros como ortofosfato ${ }^{16}$ e nitrato de amônio ${ }^{17}$.

\section{O PROBLEMA DA CONTAMINAÇÃO}

O problema da contaminação já existe a partir da coleta da amostra em função do tipo de recipiente para a armazenagem, limpeza do mesmo e da presença de partículas de poeira no ar. Às amostras de sangue acrescentem-se ainda traços de alumínio provenientes da pele do paciente, da utilização de agulhas e seringas e, quando necessário, da utilização de agentes anticoagulantes.

Oster e Prellwitz ${ }^{18}$, em sua revisão sobre o assunto, pro- 
põem uma série de medidas a serem adotadas na análise de alumínio no sangue, desde a coleta até a determinação propriamente dita. Os autores chegam a sugerir que os "primeiros mililitros" de sangue retirados dos pacientes sejam desprezados a fim de evitar contaminação.

De acordo com Savory ${ }^{19}$, os maiores problemas da determinação de alumínio a nível biológico estão nos métodos imprecisos e na contaminação. A grande imprecisão dos métodos está relacionada diretamente à baixa concentração do analito somada à extrema complexidade das matrizes, seja pela elevada concentração salina no caso dos fluidos de hemodiálise ou pelo elevado número de constituintes no caso do sangue.

Mesmo a determinação de alumínio apenas na água utilizada no preparo da solução de diálise não encontra a exatidão desejada, como mostra uma publicação na revista Clinical Chemistry $^{20}$, sobre uma análise interlaboratorial realizada durante cinco anos entre 56 laboratórios. Cada laboratório recebeu mensalmente duas amostras de fluido para diálise e duas amostras de água que continham quantidades conhecidas de alumínio. Os resultados apresentaram, para concentrações de alumínio de até $30 \mu \mathrm{g} /$ 1, desvios padrões relativos entre $45,1 \%$ e $92,3 \%$ para o fluido e entre $35,2 \%$ e $128,6 \%$ para a água. $\mathrm{Na}$ opinião dos autores, resultados tão pouco satisfatórios podem ser atribuídos à interferências da matriz e à ausência de materiais de referência. Em um outro estudo ${ }^{21}$ da mesma natureza, o teor de alumínio no soro sangüíneo foi determinado por um conjunto de laboratórios italianos por um período de sete anos (1983/1990). Segundo os autores, uma parcela muito pequena dos laboratórios $(9,1 \%)$ apresentou resultados satisfatórios e, ainda assim, não de maneira constante ao longo do tempo em que o estudo foi realizado. Eles atribuem à contaminação a grande dificuldade em analisar baixas concentrações de alumínio.

\section{FONTES DE CONTAMINAÇÃO}

As principais fontes de contaminação são recipientes, reagentes e partículas provenientes do ar. Desta forma a determinação de traços de alumínio começa pela escolha do material adequado e sua limpeza, utilização de reagentes de qualidade ou pré-purificados e a execução da análise em ambiente livre de partículas de poeira.

Recipientes de vidro são contra-indicados devido à presença do óxido de alumínio. Recipientes plásticos devem ser submetidos a uma descontaminação por ação de uma solução de lavagem. As mais empregadas são soluções de ácido nítrico $9,10,16,23$, ácido clorídrico ${ }^{20,24}$ e agentes complexantes como o EDTA ${ }^{22,25}$ para retirar os traços de alumínio das paredes dos recipientes.

Um ambiente livre de partículas de poeira é obtido através da utilização de filtros de ar, instalados de forma a purificar o ar de uma sala ou de um ambiente menor como uma capela. Isto é conseguido através da utilização de filtros HEPA (High Efficiency Particulate Air filter) desenvolvidos nos Estados Unidos durante a II Guerra Mundial para conter partículas radioativas ${ }^{26}$. Adequados para laboratórios de análise de traços são ambientes Classe 100, que devem, teoricamente, ter uma atmosfera com no máximo 100 partículas de $0,5 \mu \mathrm{m}$ por $0,028 \mathrm{~m}^{3}\left(1 \mathrm{ft}^{3}\right)^{27}$. De acordo com Howard e Statham ${ }^{28}$ os filtros HEPA tem uma eficiência maior do que $99,97 \%$ na remoção de partículas com diâmetro igual ou maior do que $0,3 \mu \mathrm{m}$.

\section{AVALIAÇÃO DA CONTAMINAÇÃO DE MATERIAIS E REAGENTES}

Os resultados aqui apresentados pretendem mostrar quão problemático e importante é o controle da contaminação na determinação de traços de alumínio, e foram obtidos durante o desenvolvimento de uma metodologia para a determinação de alumínio em concentrados salinos para diálise e soro sangüíneo.
As análises foram realizadas por AAS, medindo diretamente o teor de alumínio nas amostras ou após uma etapa de pré-concentração ${ }^{29^{*}}$.

A tabela 1 mostra os resultados encontrados na extração de alumínio de recipientes plásticos para armazenagem de amostras, com diferentes soluções de extração. Pode-se observar que tanto o ácido nítrico quanto o EDTA são bons agentes para a lavagem de recipientes plásticos. A ação do ácido nítrico é mais efetiva em soluções alcoólicas do que em soluções aquosas, provavelmente por essas molharem a superfície do plástico.

Tabela 1. Alumínio extraído de frascos para armazenar soluções, com diferentes soluções de extração (48 h de contato), em temperatura ambiente e sob agitação.

\begin{tabular}{ccc}
\hline $\begin{array}{c}\text { Tipo de } \\
\text { plástico* }\end{array}$ & $\begin{array}{c}\text { Solução } \\
\text { de extração }\end{array}$ & $\begin{array}{c}\text { Alumínio } \\
\text { extraído }\end{array}$ \\
\hline 1 & $\begin{array}{c}0,2 \% \mathrm{HNO}_{3}(\mathrm{v} / \mathrm{v}) \\
\text { sol. aquosa }\end{array}$ & $2 \mu \mathrm{g} / \mathrm{l}$ \\
1 & $\begin{array}{c}0,1 \mathrm{~mol} / \mathrm{l} \text { EDTA } \\
\text { sol. aquosa }\end{array}$ & $16 \mu \mathrm{g} / \mathrm{l}$ \\
1 & $\begin{array}{c}10 \% \mathrm{HNO}_{3}(\mathrm{v} / \mathrm{v}) \mathrm{em} \\
\mathrm{EtOH}^{*} / \mathrm{H}_{2} \mathrm{O} 1: 1\end{array}$ & $9 \mu \mathrm{g} / \mathrm{l}$ \\
1 & $\begin{array}{c}10 \% \mathrm{HNO}_{3}(\mathrm{v} / \mathrm{v}) \\
\text { sol. etanólica } \\
10 \% \mathrm{HNO}_{3}(\mathrm{v} / \mathrm{v})\end{array}$ & $20 \mu \mathrm{g} / \mathrm{l}$ \\
& sol. etanólica & $26 \mu \mathrm{g} / \mathrm{l}$ \\
\hline
\end{tabular}

* 1: Polietileno de alta densidade, marca Zinsser Polyvials, $20 \mathrm{ml}$ capacidade; 2: Polietileno de baixa densidade, frasco comum, $50 \mathrm{ml}$ capacidade.

** EtOH: Etanol.

Na tabela 2 estão os teores de alumínio extraídos de alguns materiais utilizando solução etanólica de $\mathrm{HNO}_{3} \quad 10 \%$. Pode-se observar que todos os materiais analisados contêm alumínio. Do vidro, como era de se esperar, a quantidade extraída é muito grande; $370 \mu \mathrm{g} \mathrm{Al} \mathrm{em} 50 \mathrm{ml}$ correspondem a 7,4 ppm, nível de contaminação muito alto para uma análise de traços. Plásticos apresentam um grau de contaminação não muito alto $1,3 \mu \mathrm{g} \mathrm{Al} / 50 \mathrm{ml}$, no recipiente analisado, mas mesmo assim, se não previamente limpos podem contribuir significativamente para um branco elevado. Apesar do sangue certamente não apresentar a mesma capacidade de extração do que soluções etanol/ $\mathrm{HNO}_{3}$, seringas e agulhas

Tabela 2. Alumínio extraído de diferentes tipos de materiais por uma solução etanólica de $\mathrm{HNO}_{3} 10 \%$ (v/v) em $48 \mathrm{~h}$ de contato, a temperatura ambiente e sob agitação.

\begin{tabular}{ccc}
\hline Material & Tipo de uso & Alumínio extraído \\
\hline Vidro & Balão volumétrico $50 \mathrm{ml}$ & $370 \mu \mathrm{g} / 50 \mathrm{ml}$ \\
Plástico & Frasco $50 \mathrm{ml}$ & $1,3 \mu \mathrm{g} / 50 \mathrm{ml}$ \\
& Seringa $10 \mathrm{ml}$ & $1,62 \mu \mathrm{g}$ \\
& Polietileno em pó (Merck) & $9,7 \mu \mathrm{g} / \mathrm{g}$ \\
& Polietileno em pó (Aldrich) & $2,2 \mu \mathrm{g} / \mathrm{g}$ \\
Metal & Agulha para injeção & $0,05 \mu \mathrm{g} / \mathrm{agulha}$ \\
\hline
\end{tabular}

* O alumínio na forma de seu complexo com cromoazurol S (CAS) é adsorvido (retido) em uma coluna de polietileno em pó (PE). Como coluna utilizou-se uma ponteira de pipeta descartável $(1000 \mu \mathrm{l})$ preenchida com aproximadamente $300 \mathrm{mg}$ de PE. Após a etapa de préconcentração o complexo Al-CAS é eluído com uma solução acidificada de etanol $\left(0,3 \% \mathrm{HNO}_{3}\right)$ e nesta solução etanólica, livre da matriz, determinado por espectrometria de absorção atômica em forno de grafite. 
Tabela 3. Teor de alumínio encontrado nos reagentes e soluções utilizadas na análise.

\begin{tabular}{|c|c|c|c|}
\hline Reagente/Solução & Fabricante & $\begin{array}{c}\text { Teor* } \\
{[\mu \mathrm{g} / \mathrm{g}][\mu \mathrm{g} / \mathrm{l}]}\end{array}$ & $\begin{array}{c}\text { Desvio Padrão Relativo } \\
(\mathrm{n}=4)[\%]\end{array}$ \\
\hline $\mathrm{HNO}_{3}$ p.a. (frasco I) & Merck & $110 \mu \mathrm{g} / 1$ & 3,5 \\
\hline $\mathrm{HNO}_{3}$ p.a. (frascoII) & Merck & $58 \mu \mathrm{g} / 1$ & 2,8 \\
\hline $\mathrm{HNO}_{3}$ Suprapur, & Merck & $110 \mu \mathrm{g} / 1$ & 4,3 \\
\hline $\mathrm{HNO}_{3}$ destilado & Merck & $24 \mu \mathrm{g} / 1$ & 2,7 \\
\hline $\mathrm{H}_{2} \mathrm{SO}_{4}$ p.a. & Merck & $375 \mu \mathrm{g} / \mathrm{l}$ & 15,3 \\
\hline Ácido tricloroacético & Reagen & $20,0 \mu \mathrm{g} / \mathrm{g}$ & 4,9 \\
\hline Ácido tricloroacético & Merck & $5,8 \mu \mathrm{g} / \mathrm{g}$ & 2,3 \\
\hline $\mathrm{Na}_{2} \mathrm{SO}_{4}$ p.a. & Reagen & $26,5 \mu \mathrm{g} / \mathrm{g}$ & 28,5 \\
\hline Tungstato de sódio & Merck & $26,5 \mu \mathrm{g} / \mathrm{g}$ & 12,9 \\
\hline Cromoazurol S & Riedel de Haen & $2,2 \mu \mathrm{g} / \mathrm{g}$ & 1,4 \\
\hline Sol. tampão succinato $0,5 \mathrm{~mol} / 1^{* *}$ & & $21 \mu \mathrm{g} / 1$ & 3,7 \\
\hline Heparina & Roche & $792 \mu \mathrm{g} / 1$ & 1,3 \\
\hline
\end{tabular}

*Teor calculado a partir da determinação por AAS em forno de grafite de soluções aquosas a $5 \%$ do respectivo reagente.

**Solução utilizadas na análise do concentrado salino (teor medido diretamente na solução).

Tabela 4. Teor de alumínio encontrado nos sais e glicose utilizados no preparo dos concentrados salinos, utilizando a metodologia descrita ${ }^{29}$

\begin{tabular}{lc}
\hline \multicolumn{1}{c}{ Sal* } & Teor $[\mu \mathrm{g} / \mathrm{g}]$ \\
\hline cloreto de potássio & 0,92 \\
cloreto de sódio & 0,39 \\
cloreto de magnésio & 3,35 \\
cloreto de cálcio & 3,16 \\
acetato de sódio & 0,10 \\
glicose & 0,25 \\
\hline
\end{tabular}

*Fabricante: Merck

podem, em maior ou menor extensão, provocar contaminações. Foram encontrados $1,62 \mu \mathrm{g} \mathrm{Al}$ por seringa de $10 \mathrm{ml}$ e $0,05 \mu \mathrm{g} \mathrm{Al}$ por agulha. Se considerarmos que o nível de Al no sangue, aceito como normal, situa-se em torno de $7 \mu \mathrm{g} / \mathrm{l}^{6}$, as contribuições da seringa e agulha podem ser muito significativas.

Nas tabelas 3 e 4 estão os teores de alumínio encontrados em diversos reagentes utilizados na análise do sangue e em sais utilizados no preparo de fluidos para diálise.

Sulfato de sódio, ácido sulfúrico, ácido tricloroacético e tungstato de sódio são utilizados como agentes desproteinizantes do sangue. A heparina é um agente anticoagulante e o ácido nítrico é muito utilizado nestas análises não somente como agente de limpeza mas como conservante (águas) e diluente nas análises por AAS.

Todos os reagentes estão contaminados por alumínio. Apesar de alguns resultados mostrados na tabela 3 apresentarem um elevado desvio padrão, é evidente a presença do alumínio. A destilação do ácido nítrico concentrado é muito mais eficiente e barata do que a utilização de produtos como o Suprapur da Merck. Talvez a eficiência de tal tipo de produto, verificada na determinação de outros metais, não se confirme para o alumínio, devido à armazenagem em recipientes de vidro.

Os resultados da tabela 4 mostram que mesmo reagentes de qualidade p.a. usados na preparação de fluidos para diálise apresentam traços de alumínio.

Durante o desenvolvimento da metodologia para a análise de concentrados para diálise, não foi dada, inicialmente, a devida importância à contaminação. Assim, a análise foi executada com a adoção gradativa das medidas para evitar contaminação, que no caso foram:

- Utilização de água destilada e posteriormente purificada em um sistema Milli-Q;

- Lavagem de todo o material por pelo menos $48 \mathrm{~h}$ em solução etanólica de $\mathrm{HNO}_{3} \quad 10 \%(\mathrm{v} / \mathrm{v})$;

- Utilização de ácido nítrico destilado;
- Extração do alumínio do polietileno em pó (adsorvente para a pré-concentração do $\mathrm{Al}$ ) por agitação de uma suspensão do mesmo em solução etanólica de $\mathrm{HNO}_{3} 10 \%(\mathrm{v} / \mathrm{v})$ por $48 \mathrm{~h} \mathrm{e}$ - Execução de todas as etapas da análise, com exceção da medida no espectrômetro de absorção atômica, em uma câmara de fluxo laminar.

Na tabela 5 estão os resultados de recuperações para soluções analíticas contendo de 0 a $80 \mu \mathrm{g} \mathrm{Al} / 1$ após a pré-concentração, obtidos sem que nenhuma medida para evitar a contaminação tivesse sido adotada. Pode-se observar um elevado teor de alumínio no branco e recuperações absolutamente não reprodutíveis. O alumínio encontrado nos efluentes não mostra relação de proporcionalidade com a concentração das soluções analíticas, sendo até mesmo nas soluções mais diluídas superior ao das soluções mais concentradas. Na figura 1 fica evidente que tais resultados são provenientes da contaminação introduzida pelo material, pelo polietileno em pó e por partículas de poeira. Pode-se observar que quanto menor a concentração de alumínio na amostra mais significativa é a contaminação. Enquanto que para as soluções com $80 \mu \mathrm{g} \mathrm{Al} / \mathrm{l}$ a contaminação eleva a recuperação em cerca de $20 \%$, nas soluções contendo $20 \mu \mathrm{g} \mathrm{Al} / 1$ ela corresponde a mais de $200 \%$ do total recuperado. Na tabela 6 estão os resultados encontrados para as mesmas soluções, mas após terem sido tomadas as precauções para evitar a contaminação.

Tabela 5. Recuperações dos conteúdos de alumínio em diferentes soluções analíticas após pré-concentração em coluna de politileno em pó do complexo Al-cromoazurol S e determinação por AAS.

\begin{tabular}{ccc}
\hline $\begin{array}{c}\text { Concentração } \\
\text { teórica de Al } \\
\text { após pré-conc. } \\
{[\mu \mathrm{g} / \mathrm{l}]}\end{array}$ & $\begin{array}{c}\text { Recuperação } \\
\text { no } \\
\text { eluato } \\
{[\mu \mathrm{g} / \mathrm{l}]}\end{array}$ & $\begin{array}{c}\text { Concentração } \\
\text { residual } \\
\text { no efluente } \\
{[\mu \mathrm{g} / \mathrm{l}]}\end{array}$ \\
\hline 0 & 23 & 4 \\
0 & 32 & 8 \\
0 & 16 & 15 \\
0 & 27 & 1 \\
10 & 33 & 8 \\
10 & 35 & 10 \\
20 & 37 & 5 \\
20 & 30 & 11 \\
40 & 53 & 6 \\
40 & 61 & 12 \\
60 & 69 & 4 \\
60 & 86 & 11 \\
80 & 83 & 15 \\
80 & 105 & 11 \\
\hline
\end{tabular}




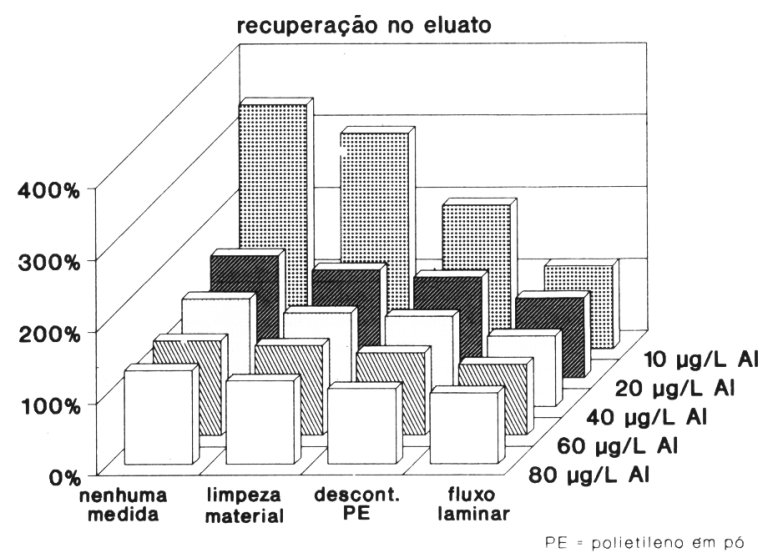

Figura 1. Influência dos fatores de contaminação na recuperação de alumínio.

Tabela 6. Recuperações dos conteúdos em alumínio $(n=3)$ de diferentes soluções analíticas, e com diferentes fatores de enriquecimento, após a introdução das medidas para descontaminação.

\begin{tabular}{rrrrr}
\hline \multicolumn{2}{c}{$\begin{array}{c}\text { Concentração } \\
\text { teórica }[\mu \mathrm{g} / \mathrm{l}] \\
\text { antes } \\
\text { após } \\
\text { pré-concentração }\end{array}$} & $\begin{array}{c}\text { Volume antes } \\
\text { da pré-conc. }[\mathrm{ml}]\end{array}$ & \multicolumn{2}{c}{ Recuperação } \\
{$[\mu \mathrm{g} / \mathrm{l}]$} & {$[\%]$} \\
\hline 0 & 0 & & & \\
0 & 0 & 25 & 0 & - \\
0 & 0 & 25 & 2 & - \\
4 & 10 & 25 & 11 & $110,0 \pm 5,3$ \\
8 & 20 & 25 & 22 & $110,0 \pm 5,4$ \\
16 & 40 & 25 & 39 & $97,5 \pm 4,7$ \\
20 & 60 & 25 & 59 & $98,3 \pm 3,3$ \\
32 & 80 & 25 & 78 & $97,5 \pm 4,2$ \\
8 & 40 & 50 & 41 & $102,5 \pm 3,4$ \\
4 & 40 & 100 & 37 & $92,0 \pm 4,4$ \\
\hline
\end{tabular}

As figuras 2 e 3 mostram o teor de alumínio encontrado em soluções com até $20 \mu \mathrm{g} / \mathrm{l} \mathrm{Al}$ antes e após as precauções com a contaminação terem sido adotadas. Pelos resultados expostos
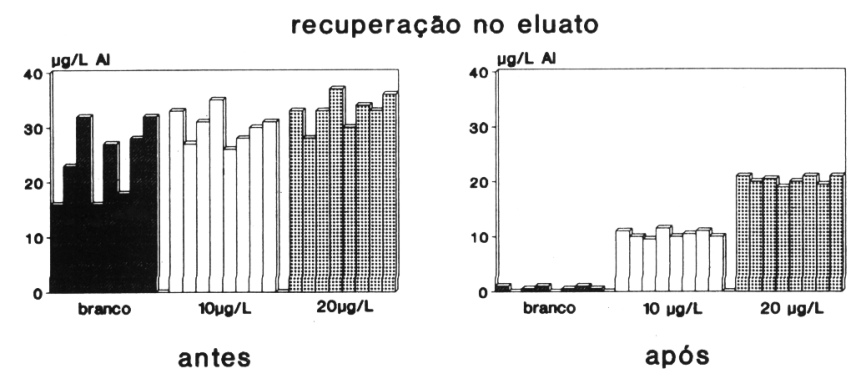

resíduo no efluente
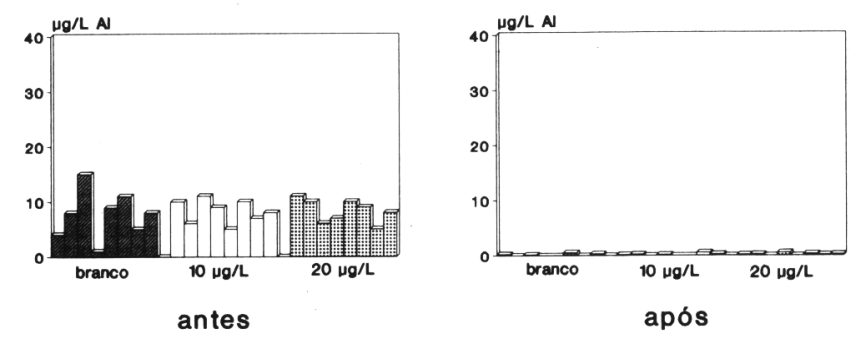

Figura 2. Recuperações do alumínio em soluções contendo até $20 \mu \mathrm{g} / \mathrm{l}$ $A l$, antes e após a adoção do conjunto de medidas para evitar a contaminação $(n=8)$.

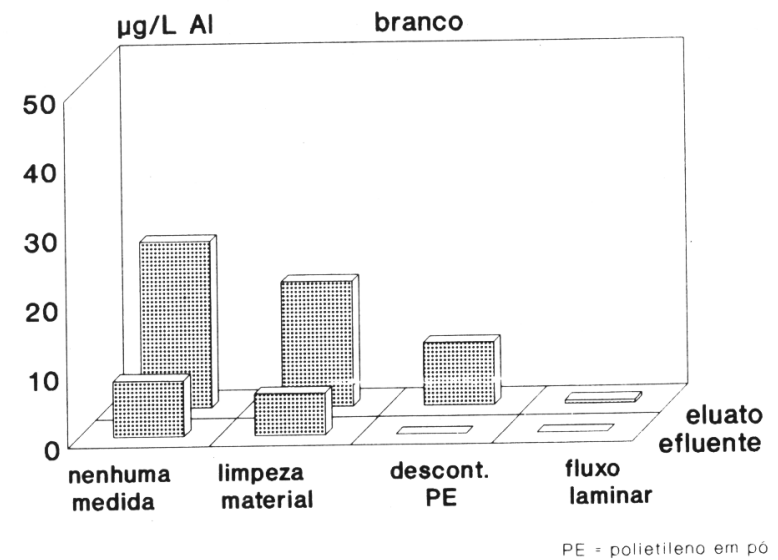

Figura 3. Influência dos fatores de contaminação na determinação de alumínio a partir de uma prova em branco.

pode-se afirmar que a determinação de traços de alumínio, na faixa de partes por bilhão, apresenta resultados satisfatórios apenas quando um conjunto de medidas para evitar a contaminação é adotado.

Somente a limpeza do material, associada ao uso de reagentes purificados ou com qualidade suficiente para apresentar reduzida contaminação e execução da análise em um ambiente livre de partículas de poeira (câmara de fluxo laminar) garantem o êxito de uma determinação de traços de alumínio.

\section{AGRADECIMENTOS}

Parte deste trabalho foi desenvolvida no Institut für Anorganische und Analytische Chemie da Technische Universität Clausthal, em Clausthal-Zellerfeld, Alemanha, com bolsa de estudos financiada pela CAPES.

\section{REFERÊRNCIAS}

1. Savory, J.; Wills, M. S.; In Metals and Their Compounds in the Environment; Merian, E., Ed.; Verlag Chemie; Weinheim 1991; p 715.

2. Warnock, D. G.; In Tratado de Medicina Interna; Cecil Ed.; 19a edição; Guanabara Koogan; Rio de Janeiro, 1993; vol. 1, p 542.

3. Alfey, A. C.; New Engl. J. Med. 1984, 310, 1113.

4. Savory, J.; Berlin, A.; J. Clin. Chem. Clin. Biochem. 1982, $20,837$.

5. Versiek, J.; Cornelis, R.; Anal. Chim. Acta 1980, 116, 217

6. Savory, J.; Wills, M. R.; CRC Crit. Rev. Clin. Lab. Sci. 1989, $27,59$.

7. Halls, D.; Fell, G. S.; Analyst 1985, 110, 243

8. Fuchs, C.; Armstrong, V. W.; Hein, H.; Kraft, B.; In Spurenelemente in der Nephrologie; Bamberg, W. S., Ed.; Dustri-Verlag; München, 1983; p 12.

9. Granadillo, V. A.; Tahán, J. E.; Salgado, O.; Elejalde, L. E.; Rodríguez-Iturbe, B.; Romero, G. B.; Romero, R. A.; Clin. Chim. Acta 1995, 233, 47.

10. De Broe, M. E.; Van Landenghem, G. F.; D'Haese, P. C.; Lamberts, L.; Anal. Chem. 1994, 66, 216.

11. Stephen, T.; Pizzolato, S.; Demshar; H. P.; J. Anal. Toxicol. 1991, 15, 66.

12. Roberts, N. B.; Birchall, J. D.; Bell, G. M.; Ahmad, R.; Fahal, I. H.; J. Anal. Atom. Spectrom. 1993, 8, 911.

13. Gutteridge, J. M. C.; Das, R. G.; Quinlan, G. J.; Redhead, K.; Pharmacol. Toxicol. 1992, 70, 278.

14. Hewitt, C. D.; Winborne, K.; Margrey, D.; Nicholson, J. R. P.; Savory, M. G.; Savory, J.; Wills, M. R.; Clin. Chem. 1990, 36, 1466. 
15. Welz, B.; Atomic Absorption Spectrometry, Verlag Chemie; Weinheim 1985; p 267.

16. Woolfson, A. D.; Gracey, G. M.; Analyst 1987, 112, 1387.

17. Smeyers-Verbeke, J. A.; Verbeelen, D.; Anal. Chem. 1988 , $60,380$.

18. Oster, O.; Prellwitz, W.; In Analytikertaschenbuch; Wisser, H. Ed.; Springer Verlag; Berlin; 1990; vol. 9, p 399.

19. Savory, J.; Wills, M. R.; Clin. Chem. 1992, 38/8, 1565.

20. Taylor, A.; Briggs, R. J.; Çevik, C.; Clin. Chem. 1994, 40/8, 1517.

21. Morisi, G.; Patriarca, M.; Menditto, A.; Microchem. J. 1992, 46, 147.
22. Pierson, K. B.; Evenson, M.; Anal. Chem. 1986, 58, 1744.

23. Reimert, S.; Andersen, J. R.; Analyst 1986, 111, 657.

24. Guillard, O.; Pineau, A.; Baruthio, F.; Arnaud, J.; Clin. Chem. 1988, 34/8, 1603.

25. Leung, F. Y.; Henderson, A. R.; At. Spectrosc. 1983, 4, 1.

26. Moody, J. R.; Anal. Chem. 1982, 54, 1358A

27. Torreira, R. P.; Salas Limpas, Hemus Editora; São Paulo 1991.

28. Howard, A. G.; Statham, P. J.; Inorganic Trace Analysis, John Wiley \& Sons; Chichester, 1993; p 21.

29. Bohrer do Nascimento, D.; Schwedt, G.; Pharmazie 1993, 48,676 . 